\title{
Next-to-leading order diphoton+2-jet production at the LHC
}

\section{Z. Bern}

Department of Physics and Astronomy, UCLA, Los Angeles, CA 90095-1547, USA

E-mail: bern@physics.ucla.edu

\section{J. Dixon}

SLAC National Accelerator Laboratory, Stanford University, Stanford, CA 94309, USA

E-mail: lanceeslac.stanford.edu

\section{F. Febres Cordero}

Departamento de Física, Universidad Simón Bolívar, Caracas 1080A, Venezuela

E-mail: ffebrescusb.ve

\section{S. Höche}

SLAC National Accelerator Laboratory, Stanford University, Stanford, CA 94309, USA

E-mail: shoechedslac.stanford.edu

\section{H. Ita}

Physikalisches Institut, Albert-Ludwigs-Universität Freiburg, D-79104 Freiburg, Germany

E-mail: harald.ita@physik.uni-freiburg.de

\section{A. Kosower}

Institut de Physique Théorique, CEA-Saclay, F-91191 Gif-sur-Yvette cedex, France

E-mail: david.kosower@cea.fr

\section{N. A. Lo Presti*}

Institut de Physique Théorique, CEA-Saclay, F-91191 Gif-sur-Yvette cedex, France

E-mail: nicola.lo-presti@cea.fr

\section{Maître}

Institute for Particle Physics Phenomenology, University of Durham, Durham DH1 3LE, UK

E-mail: daniel.maitre@durham.ac.uk

We present results from a recent calculation of prompt photon-pair production in association with two jets to next-to-leading order (NLO) at the LHC. The virtual contribution is evaluated using the BLACKHAT library, a numerical implementation of on-shell methods for one-loop amplitudes, in conjunction with SHERPA. We study four sets of cuts: standard jet cuts, a set of Higgs-related cuts suggested by ATLAS, and corresponding sets which isolate the kinematic region where the process becomes the largest background to Higgs production via vector-boson fusion.

11th International Symposium on Radiative Corrections (Applications of Quantum Field Theory to Phenomenology) (RADCOR 2013),

22-27 September 2013

Lumley Castle Hotel, Durham, UK

\footnotetext{
*Speaker.
} 


\section{Introduction}

The QCD production of a photon pair in association with jets is an important process at hadron colliders. Photon pairs are one of the key decay channels for detecting and measuring the Higgslike boson [1,2] announced last year. Accordingly, a good understanding of prompt photon-pair production is important to precision measurements of its properties and thence to exploring deviations from Standard Model expectations. In particular, when the photon pair is produced in association with two hadronic jets, the process is an important background to Higgs-like boson production via vector-boson fusion (VBF). Leading-order (LO) predictions in QCD suffer from a strong dependence on the unphysical renormalization and factorization scales. Next-to-leading order (NLO) predictions generally reduce this dependence, and offer the first quantitatively reliable predictions in perturbation theory. Inclusive diphoton production at hadron colliders was computed long ago at NLO [3, 4, 5] and even beyond [6]. The production of a photon pair in association with a single jet has also previously been calculated at NLO [7, 8]. Here we present predictions for inclusive diphoton production in association with two jets at NLO, which has also been studied recently by Gehrmann, Greiner, and Heinrich [9].

\section{2. $\gamma \gamma+2$ jets at NLO}

The ingredients that enter a NLO calculation are the Born cross section, the virtual (one-loop) corrections, and the radiative (real-emission) corrections. The latter are computed from tree-level matrix elements with an additional parton in the final state compared to the Born process. In order to implement in a numerical setting the cancellation of the separate divergences that arise in the virtual and real corrections, we use the Catani-Seymor dipole subtraction scheme [10], which introduces a fourth, subtraction, contribution to the evaluation of the NLO cross section

$$
\sigma_{n}^{\mathrm{NLO}}=\int_{n} \sigma_{n}^{\mathrm{born}}+\int_{n} \sigma_{n}^{\mathrm{virt}}+\int_{n} \Sigma_{n}^{\mathrm{subtr}}+\int_{n+1}\left(\sigma_{n+1}^{\mathrm{real}}-\sigma_{n+1}^{\mathrm{subtr}}\right) .
$$

We use the Frixione photon isolation criterion [11], which avoids the need for fragmentation contributions.

We use the SHERPA package [12] to manage the partonic subprocesses, to integrate over phase space, and to output ROOT [13] $n$-tuples. For the computation of the Born and real-emission matrix elements along with the Catani-Seymour dipole subtraction terms, we used the COMIX library [14], which is included in the SHERPA framework. It is based on a color-dressed form [15] of the Berends-Giele recursion relations [16].

We use the BlackHAT software library $[17,18]$ to evaluate the virtual contribution. This library has previously been used to evaluate the virtual contributions of a number of other LHC processes of interest, such as $W, Z / \gamma^{*}+3$ - and 4-jet production [19, 20, 21, 22], four-jet production [23], as well as in investigations of high- $p_{\mathrm{T}} W$ polarization [24], and for a study of $\gamma+n$-jet to $Z+n$-jet ratios $[25,26]$.

The techniques implemented numerically in the BLACKHAT library are known as on-shell methods, and are reviewed in refs. [27] (for other numerical implementations of on-shell methods see refs. [28, 29, 30]; for other recent developments see refs. [31]). One-loop amplitudes in QCD with massless quarks may be expressed as a sum over three different types of Feynman integrals 
(boxes, triangles, and bubbles) with additional rational terms. Since the integrals are universal and well-tabulated, the aim of the calculation is to compute their coefficients, along with the rational terms. In an on-shell approach, the integral coefficients may be computed using four-dimensional generalized unitarity $[32,33,34]$, while the rational terms may be computed either by a loop-level version [35] of on-shell recursion [36] or using $D$-dimensional unitarity [37]. We use a numerical version [17] of Forde's method [38] for the integral coefficients, and subtract box and triangle integrands in a fashion similar to the Ossola-Papadopoulos-Pittau procedure [39], in order to improve the numerical stability of the calculation. To compute the rational terms, we use a numerical implementation of Badger's massive-integrals method [40], related to $D$-dimensional unitarity.

A BLACKHAT numerical calculation of the virtual contribution proceeds as follows: it calculates the fundamental building blocks (the so-called primitive amplitudes [41]); assembles them into color-ordered amplitudes; and finally computes the interference between tree and one-loop amplitudes, multiplied by the appropriate color factors. In the results reported here, we drop the subleading-color virtual terms; we have checked that they amount to under $2 \%$ of the total cross section. The five light quarks $(u, d, c, s, b)$ are all treated as massless; we neglect contributions from real or virtual top quarks. In this calculation we include the 'pure-gluonic' contribution $g g \rightarrow \gamma \gamma g g$. This contribution is nominally of higher order, but the analogous contributions have typically been included for photon-pair production without jets or in association with one jet, because there is no corresponding tree-level gluon-initiated process, and because the large value of the gluon distribution can compensate for the contribution's additional powers of the strong coupling $\alpha_{s}$. With two associated jets, there $i s$ a tree-level process $(g g \rightarrow q \bar{q} \gamma \gamma)$, and we would expect the 'pure-gluonic' contribution to be suppressed. This is indeed what we find; it contributes less than $3 \%$ of the total cross section at NLO.

We have performed a number of checks on the virtual contributions for photon-pair production in the BLACKHAT library. We have checked their collinear factorization properties. We have checked the matrix elements at individual phase-space points against HELAC [30] and MCFM [42] values for $\gamma \gamma+0$-jet production; against GoSam [29] for $\gamma \gamma+1$-jet production; and against a previous analytic calculation (relying on [43]) for the specific subprocess $q g \rightarrow \gamma \gamma q$. We compared selected helicity configurations at individual phase-space points against GoSam for $\gamma \gamma+2$-jet production. We have also checked integrated results against MCFM for $\gamma \gamma+0$-jet production, and against Gehrmann, Greiner, and Heinrich's results [8] for $\gamma \gamma+1$-jet production.

\section{Kinematics and Observables}

In our study, we consider the inclusive process $p p \rightarrow \gamma \gamma+2$ jets at an LHC center-of-mass energy of $\sqrt{s}=8 \mathrm{TeV}$, applying the following cuts:

$$
\begin{array}{lll}
p_{\mathrm{T}}^{\gamma_{1}}>50 \mathrm{GeV}, & p_{\mathrm{T}}^{\gamma_{2}}>25 \mathrm{GeV}, & \left|\eta^{\gamma}\right|<2.5, \\
p_{\mathrm{T}}^{\text {jet }_{1}}>40 \mathrm{GeV}, & p_{\mathrm{T}}^{\text {jet }_{2}}>25 \mathrm{GeV}, & \left|\eta^{\text {jet }}\right|<4.5, \quad R_{\gamma, \text { jet }}>0.4 .
\end{array}
$$

In these expressions, $R$ is the usual boost-invariant angular distance, $R_{a b}=\left[\Delta \phi_{a b}^{2}+\Delta \eta_{a b}^{2}\right]^{1 / 2}$. We define jets using the anti- $k_{T}$ algorithm [44] with parameter $R=0.4$. The jets are ordered in $p_{\mathrm{T}}$, and are labeled $i, j=1,2, \ldots$ in order of decreasing transverse momentum $p_{\mathrm{T}}$, with jet 1 being the leading (hardest) jet. 
In addition, we also consider further cuts, which select the kinematic region for VBF production of the Higgs-like boson, with the boson decaying into two photons. We will call these the VBF cuts,

$$
M_{j j}>400 \mathrm{GeV}, \quad\left|\Delta \eta_{j j}\right|>2.8,
$$

where $M_{j j}$ is the invariant mass of the subsystem made up of the two hardest jets, and $\Delta \eta_{j j}$ is the difference in pseudorapidity between the hardest and the second-hardest jets. We will show distributions both with and without VBF cuts.

We use $\mu_{R}=\mu_{F}=\hat{H}_{\mathrm{T}} / 2$, where

$$
\hat{H}_{\mathrm{T}} \equiv p_{\mathrm{T}}^{\gamma_{1}}+p_{\mathrm{T}}^{\gamma_{2}}+\sum_{m} p_{\mathrm{T}}^{m}
$$

for the central renormalization and factorization scale in our calculation. The sum runs over all final-state partons $m$, whether or not they are inside jets that pass the cuts. This means that modifications to the cuts will not affect the value of the matrix element at a given point in phase space. This avoids unwanted dependence on experimental cuts. We quote scale variation bands corresponding to varying the scales simultaneously up and down by a factor of two, taking the maximum and minimum of differential cross sections at the five scales $\hat{H}_{\mathrm{T}} / 2 \times(1 / 2,1 / \sqrt{2}, 1, \sqrt{2}, 2)$.

The calculation proceeds in two phases: generation of $n$-tuples, and analysis. In the first phase, we generate two sets of ROOT [13] format $n$-tuples using a looser set of cuts,

$$
\begin{array}{lll}
p_{\mathrm{T}}^{\gamma_{1}}>25 \mathrm{GeV}, & p_{\mathrm{T}}^{\gamma_{2}}>25 \mathrm{GeV}, & \left|\eta^{\gamma}\right|<4.5, \\
p_{\mathrm{T}}^{\text {jet }_{1}}>25 \mathrm{GeV}, & p_{\mathrm{T}}^{\text {jet }_{2}}>25 \mathrm{GeV} .
\end{array}
$$

with a second set also imposing VBF cuts looser than those of eq. (3.2),

$$
M_{j j}>300 \mathrm{GeV}, \quad\left|\Delta \eta_{j j}\right|>2.0 .
$$

A second set of $n$-tuples is essential to obtaining reasonable statistical uncertainties for the latter cuts.

We also study the effect of an additional set of cuts, suggested by the ATLAS collaboration, which select a window on the diphoton invariant mass centered around the Higgs-like boson mass,

$$
\begin{array}{lll}
p_{\mathrm{T}}^{\gamma_{1}}>0.35 m_{\gamma \gamma}, & p_{\mathrm{T}}^{\gamma_{2}}>0.25 m_{\gamma \gamma}, & \left|y^{\gamma}\right|<2.37, \\
p_{\mathrm{T}}^{\text {jet }}>30 \mathrm{GeV}, & R_{\gamma, \text { jet }}>0.4, & \left|y^{\text {jet }}\right|<4.4, \quad 122 \leq m_{\gamma \gamma} \leq 130 .
\end{array}
$$

The additional VBF cuts here are the same as those in eq. (3.5).

As mentioned above, the $n$-tuples store intermediate results such as parton momenta and coefficients associated with the event weights for the events passing the looser cuts. In the second, analysis, phase of our calculation we impose the cuts of eq. (3.1), and in addition those of eq. (3.2) on the second set. (Alternatively, we impose the cuts of eq. (3.6), and in addition those of eq. (3.5) in a parallel set.) We compute the total cross section as well as various distributions. The $n$-tuples can be used to study the effects of varying the parton distributions, scale choices, and experimental cuts. 


\begin{tabular}{||c||c|c|c||}
\hline Cuts & LO & NLO & $g g \rightarrow \gamma \gamma g g$ \\
\hline Basic & $2.678(0.003)_{-0.577}^{+0.808}$ & $3.23(0.03)_{-0.36}^{+0.31}$ & $0.0509(0.0007)$ \\
\hline VBF & $0.1398(0.0003)_{-0.0359}^{+0.0541}$ & $0.159(0.002)_{-0.021}^{+0.016}$ & $0.004(0.001)$ \\
\hline ATLAS & $0.0886(0.0005)_{-0.0189}^{+0.0264}$ & $0.099(0.002)_{-0.010}^{+0.007}$ & $0.00157(0.00003)$ \\
\hline ATLAS VBF & $0.00392(0.00004)_{-0.00101}^{+0.00153}$ & $0.0046(0.0001)_{-0.0006}^{+0.0006}$ & $8.9(0.4) \cdot 10^{-5}$ \\
\hline
\end{tabular}

Table 1: Total cross sections in picobarns for $\gamma \gamma+2$-jet production with various sets of cuts: basic (eq. (3.1)), VBF (eqs. (3.1) and (3.2)), ATLAS (eq. (3.6)), and ATLAS VBF (eqs. (3.6) and (3.5)). The numerical integration uncertainty is given in parentheses, and the scale dependence is quoted in superscripts and subscripts. The contributions for the $g g \rightarrow \gamma \gamma g g$ subprocess, shown in the last column, are included in the NLO value.

From an experimental point of view, photons must be isolated from hadronic radiation. From a theoretical point of view, we cannot isolate them completely — for example, by excluding all radiation from a cone surrounding the photon axis - because this would disturb the cancellation of infrared singularities between virtual and real-emission contributions. The Frixione isolation criterion [11] offers a compromise between the two requirements that, unlike other isolation criteria, requires no additional non-perturbative input in the form of fragmentation functions. The Frixione isolation cone depends on the radius $\delta=\left[\left(\phi-\phi_{\gamma}\right)^{2}+\left(\eta-\eta_{\gamma}\right)^{2}\right]^{1 / 2}$ of a cone surrounding a photon, and fixes a distance-dependent limit $E(\delta)$ on the transverse hadronic energy allowed inside,

$$
\sum_{p} E_{\mathrm{T} p} \theta\left(\delta-R_{p \gamma}\right) \leq E(\delta) \quad \text { with } \quad E(\delta)=E_{\mathrm{T}}^{\gamma} \varepsilon\left(\frac{1-\cos \delta}{1-\cos \delta_{0}}\right)^{n}
$$

with the sum taken over all partons. In our calculation, we used $\varepsilon=0.5, \delta_{0}=0.4$ and $n=1$ for the Frixione isolation parameters. While the Frixione isolation does not match the experimental photon isolation, the large subtractions performed in LHC analyses to eliminate pile-up effects mean that the traditional cone isolation does not match experimental practice very well either.

In our study, we use the MSTW2008 LO and NLO PDFs [45] at the respective orders. We use the zero-momentum-squared value, $\alpha_{\mathrm{EM}}(0)=1 / 137.036$, for the electromagnetic coupling. We use the five-flavor running $\alpha_{s}(\mu)$ and the value of $\alpha_{s}\left(M_{Z}\right)$ supplied with the parton distribution functions. We do not apply corrections due to non-perturbative effects such as those induced by the underlying event or hadronization. For comparisons to experimental data it is important to incorporate these effects.

\section{Results}

Our results for the total cross sections with the basic cuts of eq. (3.1); with VBF cuts of eq. (3.2) in addition; or with the ATLAS cuts of eq. (3.6); and with the VBF cuts of eq. (3.5) in addition, are shown in table 1.

In Fig. 1, we show the transverse-momentum distribution of the leading jet, using the cuts in eq. (3.1), both before and after the VBF cuts of eq. (3.2). The general features of this distribution before VBF cuts are familiar from studies of $W$ or $Z$ production in association with jets: the rapidly- 

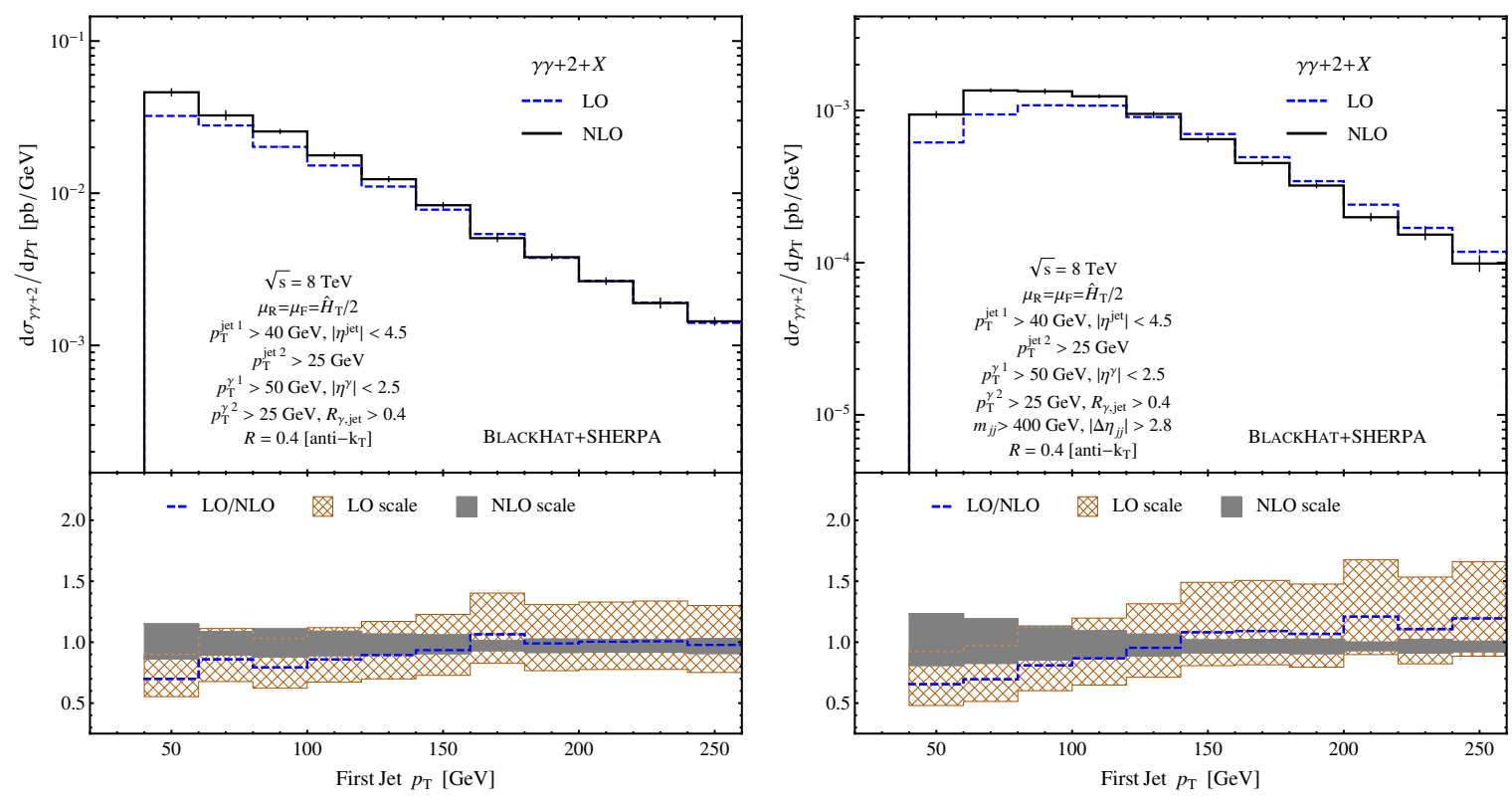

Figure 1: The leading jet's transverse-momentum distribution without (left) and with (right) VBF cuts. The upper panels show the LO (dashed blue) and NLO (solid black) predictions, while the lower panels show the ratio of these predictions to the NLO prediction, along with the LO (hatched brown) and NLO (gray) scale uncertainty bands.

falling distribution is slightly steeper at NLO than at LO, overall the NLO corrections are modest, and the scale-dependence bands narrow signficantly at NLO compared to LO.
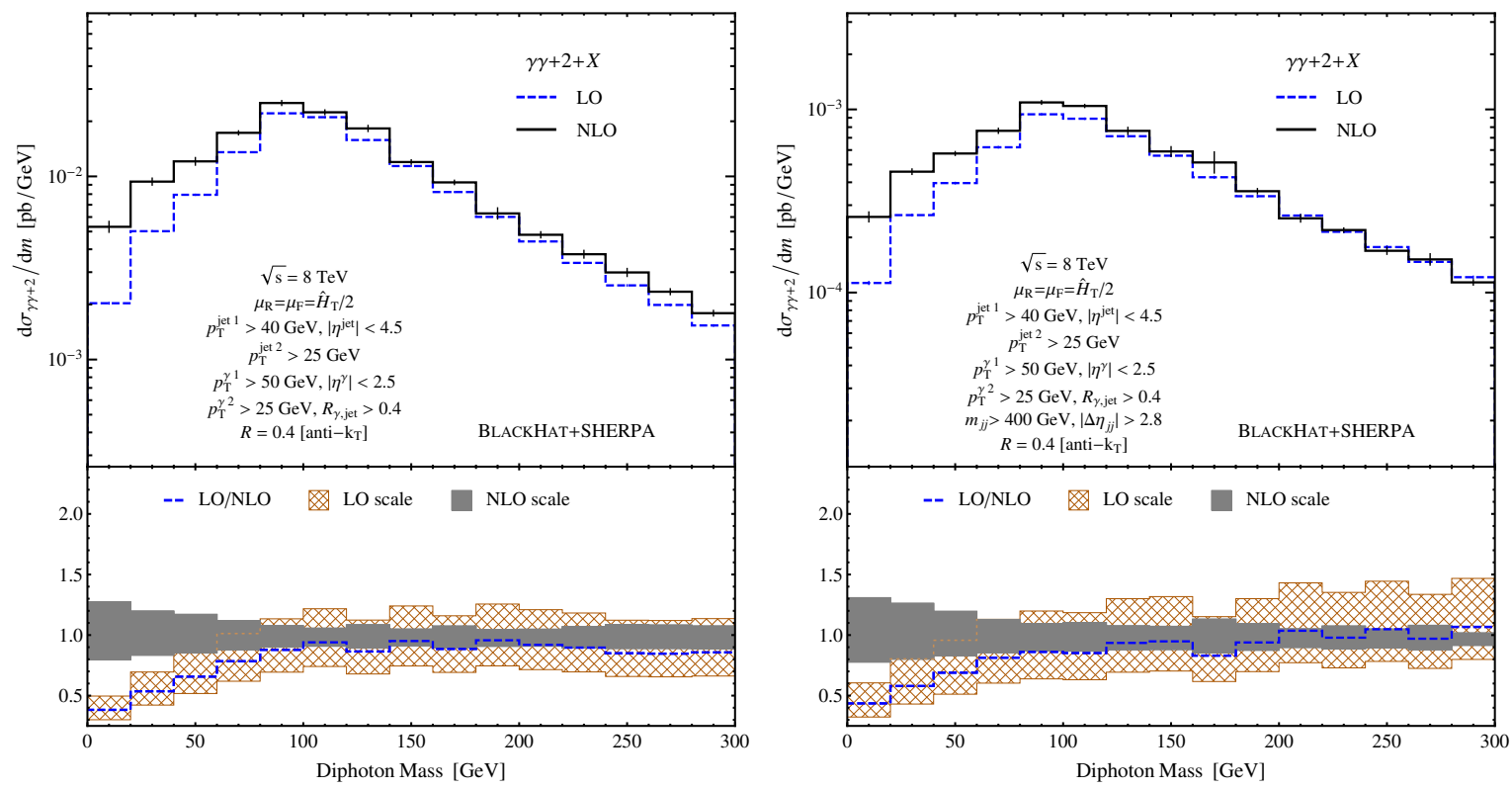

Figure 2: The diphoton invariant mass distribution without (left) and with (right) VBF cuts. The curves and bands are as in Fig. 1. 
In Fig. 2, we show the diphoton invariant mass, using the same cuts. In these distributions, and also the leading-jet $p_{\mathrm{T}}$ distribution after VBF cuts, we see that there are sizeable NLO corrections at low values of the observable. These are presumably due to relaxation of kinematic constraints with additional radiation; the large corrections are then accompanied by a widening of the scale-dependence band, as the additional power of the strong coupling will cause the real-radiation contribution alone to have larger scale dependence than the Born contribution. The NLO scale dependence bands widen slightly at large diphoton invariant mass, though this effect is eliminated by the VBF cuts.
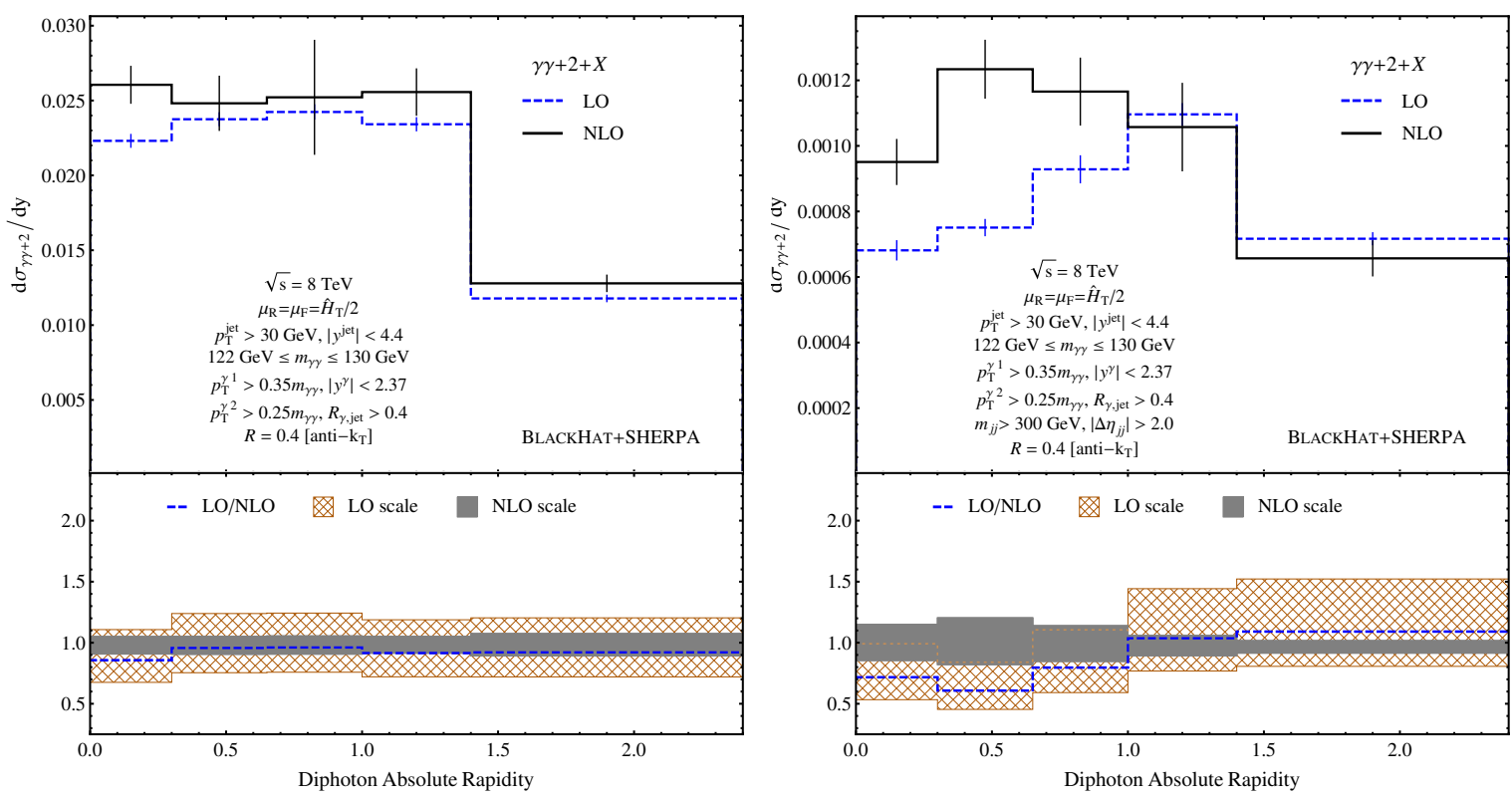

Figure 3: The distribution of the absolute value of the diphoton rapidity, using ATLAS-suggested cuts, without (left) and with (right) VBF cuts. The curves and bands are as in Fig. 1.

In Fig. 3, we show the distribution of the absolute value of the diphoton rapidity, using the cuts in eq. (3.6), both before and after the VBF cuts of eq. (3.5). In Fig. 4, we show the distribution of the azimuthal angle between the two leading jets, using the same cuts. The NLO corrections to both distributions are modest before VBF cuts, with the NLO corrections favoring emission which allows the two jets to come closer in azimuthal angle. Here, the VBF cuts do result in noticeable (to diphoton rapidity) or large (to dijet angular difference) corrections, tending to enhance contributions with central rapidity for the diphoton pair, and with small azimuthal separation between the leading jets. In both regions, the NLO scale dependence is correspondingly larger.

In this contribution, we have presented a computation of $\gamma \gamma+2$-jet production at NLO in QCD. We have given results for the total cross section and a number of distributions, under a variety of cuts. The sets of cuts include those which isolate kinematic regions relevant to vector-boson fusion production of the Higgs-like boson, to which the process we have studied is an important background. We leave a number of interesting issues to future studies. 

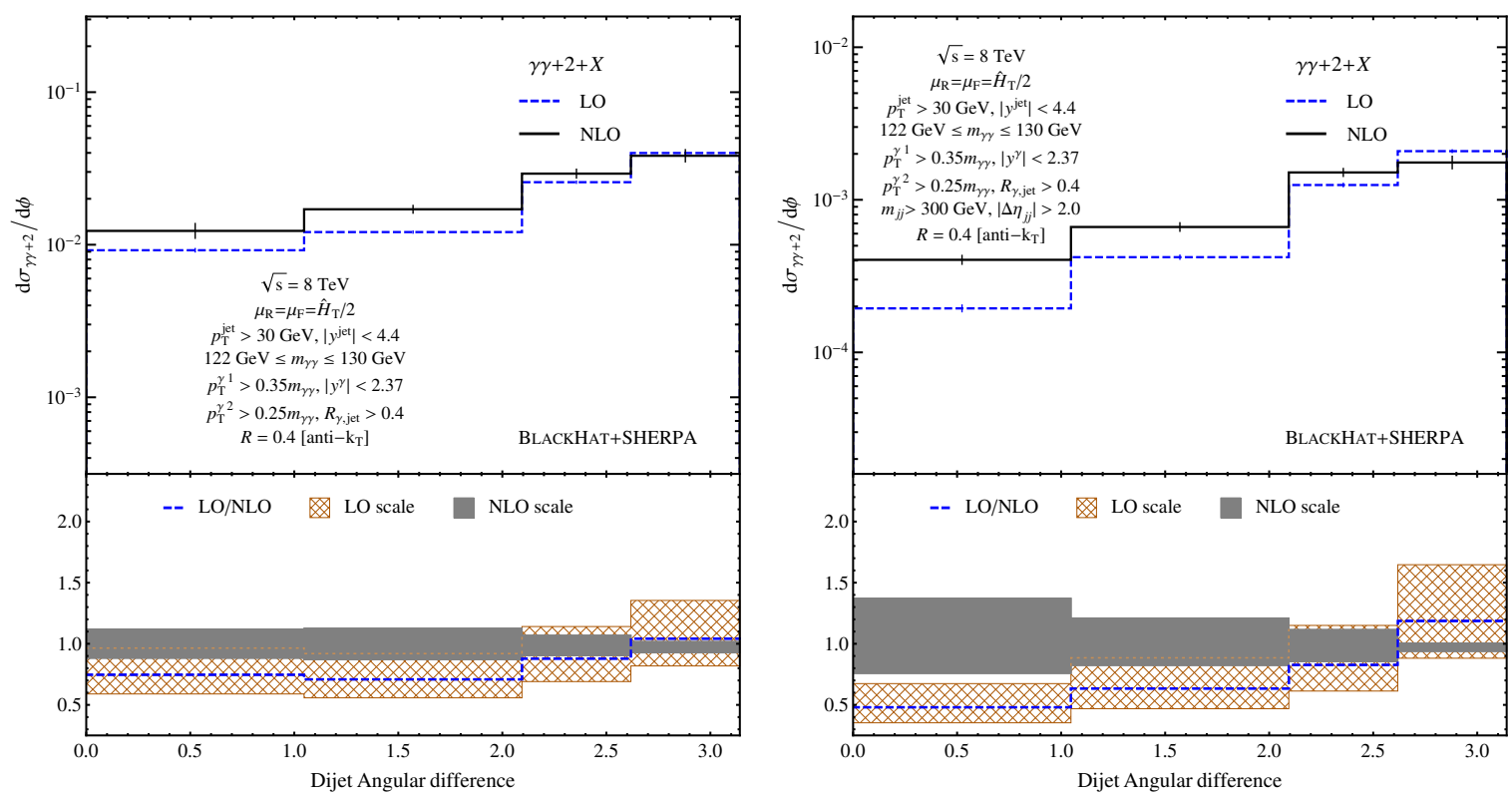

Figure 4: The distribution of the azimuthal angle difference between the two hardest jets, using ATLASsuggested cuts, without (left) and with (right) VBF cuts. The curves and bands are as in Fig. 1.

\section{Acknowledgments}

We thank Joey Huston for helpful discussions. This research was supported by the US Department of Energy under contracts DE-SC0009937 and DE-AC02-76SF00515. DAK and NALP's research is supported by the European Research Council under Advanced Investigator Grant ERCAdG-228301. DM's work was supported by the Research Executive Agency (REA) of the European Union under the Grant Agreement number PITN-GA-2010-264564 (LHCPhenoNet). This research used resources of Academic Technology Services at UCLA.

\section{References}

[1] G. Aad et al. [ATLAS Collaboration], Phys. Lett. B 716, 1 (2012) [arXiv:1207.7214 [hep-ex]].

[2] S. Chatrchyan et al. [CMS Collaboration], Phys. Lett. B 716, 30 (2012) [arXiv:1207.7235 [hep-ex]].

[3] T. Binoth, J. P. Guillet, E. Pilon and M. Werlen, Eur. Phys. J. C 16 (2000) 311 [hep-ph/9911340].

[4] Z. Bern, L. J. Dixon and C. Schmidt, Phys. Rev. D 66 (2002) 074018 [hep-ph/0206194].

[5] J. M. Campbell, R. K. Ellis and C. Williams, JHEP 1107, 018 (2011) [arXiv:1105.0020 [hep-ph]].

[6] S. Catani, L. Cieri, D. de Florian, G. Ferrera and M. Grazzini, Phys. Rev. Lett. 108 (2012) 072001 [arXiv:1110.2375 [hep-ph]].

[7] V. Del Duca, F. Maltoni, Z. Nagy and Z. Trócsányi, JHEP 0304 (2003) 059 [hep-ph/0303012].

[8] T. Gehrmann, N. Greiner and G. Heinrich, JHEP 1306 (2013) 058 [arXiv:1303.0824 [hep-ph]].

[9] T. Gehrmann, N. Greiner and G. Heinrich, arXiv:1308.3660 [hep-ph]; arXiv:1311.4754, in these proceedings. 
[10] S. Catani and M. H. Seymour, Nucl. Phys. B 485, 291 (1997) [Erratum-ibid. B 510, 503 (1998)] [hep-ph/9605323].

[11] S. Frixione, Phys. Lett. B 429 (1998) 369 [hep-ph/9801442].

[12] T. Gleisberg, S. Höche, F. Krauss, A. Schälicke, S. Schumann and J. C. Winter, JHEP 0402, 056 (2004) [hep-ph/0311263]; T. Gleisberg, S. Höche, F. Krauss, M. Schönherr, S. Schumann, F. Siegert and J. Winter, JHEP 0902, 007 (2009) [arXiv:0811.4622 [hep-ph]].

[13] R. Brun and F. Rademakers, Nucl. Instrum. Meth. A 389, 81 (1997).

[14] T. Gleisberg and S. Höche, JHEP 0812, 039 (2008) [arXiv:0808.3674 [hep-ph]].

[15] C. Duhr, S. Höche and F. Maltoni, JHEP 0608, 062 (2006) [hep-ph/0607057].

[16] F. A. Berends and W. T. Giele, Nucl. Phys. B 306, 759 (1988).

[17] C. F. Berger, Z. Bern, L. J. Dixon, F. Febres Cordero, D. Forde, H. Ita, D. A. Kosower and D. Maître, Phys. Rev. D 78, 036003 (2008) [arXiv:0803.4180 [hep-ph]].

[18] C. F. Berger, Z. Bern, L. J. Dixon, F. Febres Cordero, D. Forde, T. Gleisberg, H. Ita, D. A. Kosower and D. Maitre, Phys. Rev. Lett. 102, 222001 (2009) [arXiv:0902.2760 [hep-ph]].

[19] C. F. Berger, Z. Bern, L. J. Dixon, F. Febres Cordero, D. Forde, T. Gleisberg, H. Ita, D. A. Kosower and D. Maître, Phys. Rev. D 80, 074036 (2009) [arXiv:0907.1984 [hep-ph]].

[20] C. F. Berger, Z. Bern, L. J. Dixon, F. Febres Cordero, D. Forde, T. Gleisberg, H. Ita, D. A. Kosower and D. Maître, Phys. Rev. D 82, 074002 (2010) [arXiv:1004.1659 [hep-ph]].

[21] C. F. Berger, Z. Bern, L. J. Dixon, F. Febres Cordero, D. Forde, T. Gleisberg, H. Ita, D. A. Kosower and D. Maître, Phys. Rev. Lett. 106, 092001 (2011) [arXiv:1009.2338 [hep-ph]].

[22] H. Ita, Z. Bern, L. J. Dixon, F. Febres Cordero, D. A. Kosower and D. Maître, Phys. Rev. D 85, 031501 (2012) [arXiv:1108.2229 [hep-ph]].

[23] Z. Bern, G. Diana, L. J. Dixon, F. Febres Cordero, S. Höche, D. A. Kosower, H. Ita, D. Maître and K. Ozeren, Phys. Rev. Lett. 109, 042001 (2012) [arXiv:1112.3940 [hep-ph]].

[24] Z. Bern, G. Diana, L. J. Dixon, F. Febres Cordero, D. Forde, T. Gleisberg, S. Höche, H. Ita, D. A. Kosower, D. Maitre and K. Ozeren, Phys. Rev. D 84, 034008 (2011) [arXiv:1103.5445 [hep-ph]].

[25] Z. Bern, G. Diana, L. J. Dixon, F. Febres Cordero, S. Höche, H. Ita, D. A. Kosower, D. Maître and K. J. Ozeren, Phys. Rev. D 84, 114002 (2011) [arXiv:1106.1423 [hep-ph]].

[26] Z. Bern, G. Diana, L. J. Dixon, F. Febres Cordero, S. Höche, H. Ita, D. A. Kosower, D. Maître and K. J. Ozeren, Phys. Rev. D 87, 034026 (2013) [arXiv:1206.6064 [hep-ph]].

[27] Z. Bern, L. J. Dixon and D. A. Kosower, Ann. Rev. Nucl. Part. Sci. 46 (1996) 109 [hep-ph/9602280]; Z. Bern, L. J. Dixon and D. A. Kosower, Annals Phys. 322 (2007) 1587 [arXiv:0704.2798 [hep-ph]]; R. Britto, J. Phys. A 44 (2011) 454006 [arXiv:1012.4493 [hep-th]]; H. Ita, J. Phys. A 44 (2011) 454005 [arXiv:1109.6527 [hep-th]]; R. K. Ellis, Z. Kunszt, K. Melnikov and G. Zanderighi, Phys. Rept. 518 (2012) 141 [arXiv:1105.4319 [hep-ph]].

[28] G. Ossola, C. G. Papadopoulos and R. Pittau, JHEP 0803, 042 (2008) [arXiv:0711.3596 [hep-ph]]; J.-C. Winter and W. T. Giele, arXiv:0902.0094 [hep-ph]; S. Badger, B. Biedermann, P. Uwer and V. Yundin, Phys. Lett. B 718, 965 (2013) [arXiv:1209.0098 [hep-ph]]; Comput. Phys. Commun. 184, 1981 (2013) [arXiv:1209.0100 [hep-ph]]. 
[29] P. Mastrolia, G. Ossola, T. Reiter and F. Tramontano, JHEP 1008, 080 (2010) [arXiv:1006.0710 [hep-ph]];

[30] G. Bevilacqua, M. Czakon, M. V. Garzelli, A. van Hameren, A. Kardos, C. G. Papadopoulos, R. Pittau and M. Worek, Comput. Phys. Commun. 184, 986 (2013) [arXiv:1110.1499 [hep-ph]]; G. Cullen, N. Greiner, G. Heinrich, G. Luisoni, P. Mastrolia, G. Ossola, T. Reiter and F. Tramontano, Eur. Phys. J. C 72, 1889 (2012) [arXiv:1111.2034 [hep-ph]];

[31] A. Bredenstein, A. Denner, S. Dittmaier and S. Pozzorini, JHEP 0808, 108 (2008) [0807.1248 [hep-ph]]; Phys. Rev. Lett. 103, 012002 (2009) [0905.0110 [hep-ph]]; JHEP 1003, 021 (2010) [1001.4006 [hep-ph]];

T. Binoth, N. Greiner, A. Guffanti, J. P. Guillet, T. Reiter and J. Reuter, Phys. Lett. B 685, 293 (2010) [0910.4379 [hep-ph]]. F. Cascioli, P. Maierhofer and S. Pozzorini, Phys. Rev. Lett. 108, 111601 (2012) [arXiv:1111.5206 [hep-ph]].

[32] Z. Bern, L. J. Dixon, D. C. Dunbar and D. A. Kosower, Nucl. Phys. B 425, 217 (1994) [hep-ph/9403226]; Nucl. Phys. B 435, 59 (1995) [hep-ph/9409265].

[33] Z. Bern, L. J. Dixon and D. A. Kosower, Nucl. Phys. B 513, 3 (1998) [hep-ph/9708239].

[34] R. Britto, F. Cachazo and B. Feng, Nucl. Phys. B 725, 275 (2005) [hep-th/0412103].

[35] C. F. Berger, Z. Bern, L. J. Dixon, D. Forde and D. A. Kosower, Phys. Rev. D 74, 036009 (2006) [hep-ph/0604195].

[36] R. Britto, F. Cachazo, B. Feng and E. Witten, Phys. Rev. Lett. 94, 181602 (2005) [hep-th/0501052].

[37] Z. Bern and A. G. Morgan, Nucl. Phys. B 467, 479 (1996) [hep-ph/9511336]; Z. Bern, L. J. Dixon, D. C. Dunbar and D. A. Kosower, Phys. Lett. B 394 (1997) 105 [hep-th/9611127]; C. Anastasiou, R. Britto, B. Feng, Z. Kunszt and P. Mastrolia, Phys. Lett. B 645 (2007) 213 [hep-ph/0609191]; R. Britto and B. Feng, JHEP 0802, 095 (2008) [arXiv:0711.4284 [hep-ph]]; W. T. Giele, Z. Kunszt and K. Melnikov, JHEP 0804, 049 (2008) [arXiv:0801.2237 [hep-ph]]; R. Britto, B. Feng and P. Mastrolia, Phys. Rev. D 78, 025031 (2008) [arXiv:0803.1989 [hep-ph]]; R. K. Ellis, W. T. Giele, Z. Kunszt and K. Melnikov, Nucl. Phys. B 822 (2009) 270 [arXiv:0806.3467 [hep-ph]].

[38] D. Forde, Phys. Rev. D 75, 125019 (2007) [arXiv:0704.1835 [hep-ph]].

[39] G. Ossola, C. G. Papadopoulos and R. Pittau, Nucl. Phys. B 763, 147 (2007) [hep-ph/0609007].

[40] S. D. Badger, JHEP 0901, 049 (2009) [arXiv:0806.4600 [hep-ph]].

[41] Z. Bern, L. J. Dixon and D. A. Kosower, Nucl. Phys. B 437, 259 (1995) [hep-ph/9409393].

[42] J. M. Campbell and R. K. Ellis, Phys. Rev. D 65, 113007 (2002) [hep-ph/0202176].

[43] Z. Bern, L. J. Dixon and D. A. Kosower, Nucl. Phys. B 437, 259 (1995) [hep-ph/9409393].

[44] M. Cacciari, G. P. Salam and G. Soyez, JHEP 0804, 063 (2008) [arXiv:0802.1189 [hep-ph]].

[45] A. D. Martin, W. J. Stirling, R. S. Thorne and G. Watt, Eur. Phys. J. C 63, 189 (2009) [arXiv:0901.0002 [hep-ph]]. 